Review

\title{
Computational Modelling of Deep Brain Stimulation for Parkinson's Disease: A Critical Review
}

Kristen W. Carlson ${ }^{1,2,}{ }^{*}$, Jay L. Shils ${ }^{3}$, Sahil Patel ${ }^{4}$, Longzhi Mei ${ }^{1,2}$, Jeffrey E. Arle ${ }^{1,2,5}$

1. Department of Neurosurgery, Beth Israel Deaconess Medical Center, Boston, MA, USA; E-Mails: kwcarlso@bidmc.harvard.edu; Imei@bidmc.harvard.edu; jarle@bidmc.harvard.edu

2. Department of Neurosurgery, Harvard Medical School, Boston, MA, USA

3. Department of Anesthesiology, Rush Medical Center, Chicago, IL, USA; E-Mail: Jay L Shils@rush.edu

4. University of California, Santa Barbara, California, USA; E-Mail: sdpatel@ucsb.edu

5. Department of Neurosurgery, Mount Auburn Hospital, Cambridge, MA, USA

* Correspondence: Kristen W. Carlson; E-Mail: kwcarlso@bidmc.harvard.edu

Academic Editor: Bart Ellenbroek

Special Issue: Deep Brain Stimulation for Neurobiology Diseases

OBM Neurobiology

2021, volume 5, issue 2

doi:10.21926/obm.neurobiol.2102095
Received: November 23, 2020

Accepted: April 30, 2021

Published: May 14,2021

\begin{abstract}
We review the use of numerical and computational models to explore deep brain stimulation for Parkinson's disease (DBS PD). It is a review for the modeler and those interested in PD DBS modelling methods and their value. The main model categories of active fiber, mean field, driving force, and volume of tissue activated are described as well as many modelling techniques. We give the basic requirements for a DBS PD model and current theories of DBS mechanism of action, PD etiology, and movement selection. The emphasis is on providing the reader with a representative sample of the variety of models and the range of techniques that have been applied to DBS PD, describing and critiquing them, and less so on study results. However, an extensive set of data and results that can be used for model calibration, validation, and comparison is provided in a Supplement.
\end{abstract}

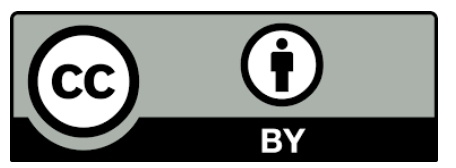

(C) 2021 by the author. This is an open access article distributed under the conditions of the Creative Commons by Attribution License, which permits unrestricted use, distribution, and reproduction in any medium or format, provided the original work is correctly cited. 


\section{Keywords}

Parkinson's disease; deep brain stimulation; numerical modelling; computational modelling; neural circuitry; connectome

\section{Glossary and Abbreviations}

Active fiber model. In contrast to a passive nerve fiber model, the electrical conductance of the fiber changes over time. Specifically, the active ion channel dynamics that regenerate an action potential along the fiber are captured at the specific ion level with characteristic gate time constants and proportion of gates open vs. closed.

Akinesia. Difficulty initiating movement; absence of intended movement.

Basal ganglia (BG). Circuitry of multiple nuclei-caudate, putamen, globus pallidus, substantia nigra, and the subthalamic nucleus-communicating with motor cortex and parts of thalamus associated with movement and reinforcement learning. Loss of cells in the substantia nigra is considered the primary seat of degradation associated with PD.

Beta band. Power spectrum of recorded neural activity that displays peaks correlated with movement disorders (MD) in PD. Low beta: 13-20 Hz. High beta; 21-35 Hz.

Bradykinesia. Slowness of movement that is a cardinal symptom of PD, including reduction of automatic movement (e.g. blinking, swinging arms, and shuffling feet while walking) and difficulty initiating movement (such as getting out of a chair).

Contraversive movement. Muscle movement on one side of the body that is controlled by the opposite hemisphere of the brain.

Cortical synchronization. Biometric of PD MD. Correlation of beta phase $(13-20 \mathrm{~Hz})$ in the BG (GPi) to broadband (50-200 Hz) gamma power in the motor cortex and hypokinetic MD. $\mathrm{Y}$-power peaks are coupled to, and precede, $\beta$-power troughs [1, 2].

Deep brain stimulation (DBS). Stimulation of brain via implanted electrodes targeted below cortical level [3-5].

Direct pathway. Inhibitory monosynaptic projection from the dopaminergic striatum D1receptor to GPi output nuclei, which then target parts of thalamus and brainstem. Facilitates voluntary movement by suppressing GPi activity that inhibits a specific action selection [6-8].

Dystonia. Involuntary muscle contractions from higher brain center malfunction, often sustained and quasi-coordinated, of which one cause can be basal ganglia dysfunction.

Fast-spiking interneuron (FSI). Although the smaller cell type in the striatum ( $5 \%)$, FSNs play a key role in motor program processing.

Freezing of Gait (FOG). Abnormal gait frequently seen in PD caused by inability to move feet while attempting to walk. Appears as quick, stepping movements in place while torso moves forward, sometimes causing a fall. 'Magnetic gait' used synonymously. Typical situations: initiating steps, approaching and navigating a destination (e.g. a doorway), turning.

Globus pallidus pars externa (GPe). Sub-component of GP receiving input from striatum and STN, and sending output to STN, GPi, and brainstem [6, 9].

Globus pallidus pars interna (GPi). Output nucleus of BG to motor thalamus, along with SNr. Suppresses unwanted action selection among competing motor programs $[6,9]$. 
High-frequency (HF) DBS. Stimulation frequency $>100 \mathrm{~Hz}$, typically $130-180 \mathrm{~Hz}$ (e.g. 130 or 150 $\mathrm{Hz})[10]$.

Hyperdirect pathway. Cortex to STN projection hypothesized to suppress inappropriate movement and perhaps fine-tune motor circuitry signaling $[6,8,11,12]$.

Indirect pathway. Inhibitory polysynaptic projection from dopaminergic striatum D2-receptor to GPe output nuclei, which then inhibits GPi output nuclei $[7,8,13]$.

Low-frequency (LF) DBS. Stimulation frequency $<100 \mathrm{~Hz}$, typically 60 or $80 \mathrm{~Hz}$ [10].

Mechanism of Action (MoA). A mechanistic cause, usually at an underlying biological systems layer such as the genome, proteome, cell, and in neurology, neural circuitry, of a disease or disorder.

Medium-spiny neuron (MSN). Predominant cell type (95\%) in the striatum, divided into two dopaminergic cells, D1 and D2, that project inhibitory connections to SNc and VTA.

Movement disorder (MD). Abnormal voluntary or involuntary movements. Three main types in PD: bradykinesia, tremor, and rigidity.

Parkinson's disease (PD). Prevalent, age-related neurological disorder (1 million US, 10 million worldwide. US cost: $\$ 52 \mathrm{~B}$ annually) characterized by depletion of dopaminergic neurons in the substantia nigra pars compacta [14]. Symptoms include rigidity, resting tremor, hyposmia, posture change, autonomic dysfunction, and cognitive deficits.

Passive fiber model. In contrast to an active nerve fiber model, the electrical properties of the fiber are constant and the active processes that regenerate the action potential along the fiber are not captured.

Resting state functional connectivity (rsfMRI). A major, evolving magnetic resonance technique to identify and analyze the functional domains in the brain, its healthy and diseased states, and the degree to which interventions restore a healthy state [15].

Resting tremor. Involuntary 4-8 $\mathrm{Hz}$ oscillation of a resting limb, suppressed by voluntary movement. Most prevalent form is a 'pill rolling' movement of the wrist in PD.

Rigidity (aka cogwheel rigidity). Muscle stiffness or restriction of movement, a cardinal PD symptom.

Striatum. Largest BG nucleus, containing 95\% inhibitory and 5\% excitatory groups, which is the primary input to the rest of the BG and to vulnerable dopaminergic nuclei in PD, the SNc and VTA. Sub-nuclei: caudate, putamen, and ventral striatum, which contains the nucleus accumbens.

Substantia nigra pars compacta (SNc). Primary region where dopaminergic neurons are lost in $\mathrm{PD}$, which is asymptomatic until progression is advanced, typically by loss of $80 \%$ of dopaminergic neurons. Processes input from cortex and projects to other BG nuclei.

Substantia nigra pars reticulata (SNr). Output nucleus of BG to thalamus and superior colliculus, mostly inhibitory (GABAergic), along with GPi. Also inhibits the substantia nigra pars compacta. Inputs are in the direct and indirect pathways from the striatum.

Subthalamic nucleus (STN). BG component that is the most common target of DBS.

Ventral tegmental area (vTA). Region secondary to SNc where dopaminergic neurons are lost in PD. Projects to striatum.

Volume of tissue activated (VTA). A primary metric of DBS efficacy: the volume of tissue in which action potentials are generated by DBS, in the STN for example. 


\section{Introduction}

In neuromodulation as a primary treatment for neurological disorders, a secondary goal has been to uncover mechanisms of action (MoA) of treatments to optimize neuromodulation efficacy [16]. Discovered in 1987, deep brain stimulation for Parkinson's disease (DBS PD), a largely reversible procedure with few side effects, was hypothesized to be an electrical 'lesion,' and later began replacing irreversible, surgical ablative procedures (lesions via thalamotomy, pallidotomy) and avoiding their side effects [17]. DBS PD has since proven efficacious to alleviate movement disorders in tens of thousands of implants and opened the door to over 20 neuromodulation treatments and brain targets for neurological disorders, notably for pharmaco-resistant patients, i.e. those for whom dopamine agonists is ineffective (see Table 1 in Udupa \& Chen [18]). Attempts to identify the underlying mechanism of DBS as an electrical 'lesion' for movement disorders revealed conceptual naiveté $[17,19]$, and were augmented by hypotheses that DBS suppressed pathological rhythms or entrained restorative rhythms [18]. Another mechanistic hypothesis is removal of an 'information block' [20, 21]. However, identifying the mechanism of action of DBS has proven difficult [22-24] and modelling studies cite this fact as part of their motivation. The causes of DBS side effects and the means to eliminate them likewise have not been fully uncovered [25]. Estimating parameters accurately is difficult in many biological settings [4], which can be offset by computational modelling $[8,26]$. One approach to address large error bars is to model parameter sweeps to see behaviour across the plausible or reported ranges of a parameter and to perform sensitivity analyses to determine which parameters affect a given result.

A desirable goal of models is being able to capture only the phenomena of interest, with the possible limitation of missing causative components. This Occam's Razor approach has evolved from experiences with complex models whose development time increases exponentially with complexity, and, especially in neural circuitry with recurrent connections, where results are difficult to decipher.

Efforts to uncover the mechanism of PD etiology now incorporate biochemistry, structural biology, molecular biology, and genetics, though to date no fully MoA-based treatment has emerged (e.g., L-dopa was originally tried for the wrong reasons) [27]. Computer modelling has assumed an increasingly important role due to many advantages such as the ability to explore phenomena inexpensively, quickly, in a large parameter space, and by capturing phenomena that would be difficult or impossible with animal or human subjects [28-30]. Numerical and computational models formalize a theory along with empirical findings incorporated into the model. Models are a test platform to explore hypotheses about the underlying MoA and predict the effects of new neuromodulation protocols on side effects and efficacy [31].

Accordingly, we describe numerical methodologies that are innovative and valuable additions to the modeler's toolbox. We include efforts toward closed-loop DBS (aka 'adaptive' or 'aDBS') due to its potential benefit to patientsand because aDBS imposes a rigid set of constraints on models that can aid successful modelling.

\subsection{Literature Search Methods}

We did a PubMed, Europe PMC, and Harvard Library Hollis system search with combinations of keywords 'deep brain stimulation, Parkinson's disease, movement disorder, circuitopathy, neuromodulation, model, computational model, and numerical model'. The inclusive criteria were: 
1) The model is an exemplar of important methods or techniques, and 2) The model is transparent and repeatable. The latter criterion did not exclude complex models or difficult mathematics but did require clarity of exposition such that methods could be understood by others and reproduced in programming code. We did not emphasize the significance of modelers' results per se.

However, in an extensive Supplement we compile qualitative and quantitative empirical findings for model calibrations or validations and salient results to reproduce or compare to modelers' own results. The article attempts to help modelers assess tools and paradigms that hold the promise of uncovering PD DBS' MoA.

To date, DBS targeting the posterior subthalamic area and thalamic intermediate nucleus are done primarily for tremor vs. the primary PD targets of STN or GPi, and as such the former targets were not included in our search [32].

\section{Brief Etiology of Parkinson's Disease}

Since DBS PD is primarily used to treat PD movement disorders (MD), we focus on MD-relevant etiology here. PD, first described by James Parkinson in 1817, is an age-related neurodegenerative disease in which motor symptoms appear to be related to loss of dopamine-expressing neurons in the substantia nigra pars compacta (SNc, 'dark substance') and to a lesser extent in the ventral tegmental area (VTA). Lesioning the SNc in monkeys results in dopamine loss in the striatum and creates a parkinsonian-like state [27]. It is estimated from a rat model that $\sim 80 \%$ of dopaminergic neurons must be lost for PD symptoms to appear, indicating a large amount of redundancy or signalto-noise ratio exists in the BG circuitry; $80 \%$ is $\sim 400,000$ of the $\sim 500,000$ dopaminergic neurons in human SNc/VTA [33]. From low levels in earlier decades, PD prevalence jumps to $15 \%$ for patients over 60 years of age and increases $15-20 \%$ in each subsequent decade, constituting $1 \%$ overall prevalence over age 60 [34].

Neuropathological changes are observed at the tissue and cellular levels: neuronal loss in the ventrolateral cell group in the substantia nigra that projects to the posterolateral putamen, with accumulation of a-synuclein (Lewy Bodies) in those cells. Dopaminergic loss is thought to be caused by accumulation of pathological alpha-synuclein or mitochondrial dysfunction, or both $[27,35]$.

The basal ganglia nuclei are strongly coupled, and effects of dopamine depletion on the striatum neural receptors are surprisingly complex and subtle [8]. Dopamine depletion correlates with increases in beta band power spectra $(13-30 \mathrm{~Hz})$ in the BG-cortical loop (see Supplement Table S4), which is also predictive of bradykinesia, and although beta band is a channel used for motor cortex to send instructions for weak-to-moderate contractions to muscle [36], a causal relationship between increased beta power and PD MDs has not been established [30]. The difficulty of sorting out cause and effect in PD etiology suggests elucidation via modelling, yet many studies seem to assume a causal relationship [37]. At the neuronal and circuit systems levels, attempts to correlate observable symptoms of PD with progressive and graded dopaminergic neuron loss are worthwhile modelling goals, as early diagnosis of PD is critical to improving pharmaceutical, electroceutical, or neuromodulation treatment efficacy as well as testing DBS PD MoA hypotheses [27].

An excellent short history of PD etiology is given by Goedert et al. [27]. Etiology of PD at the genetic signaling pathway level is summarized in the Supplement. 


\section{Theory of Movement Selection by Basal Ganglia and PD Etiology}

Hypotheses on the MoA in PD MD center on the complex interaction within the BG, and between the BG, thalamus, and cortex (Figure 1). Modelers focus on three main signaling pathways that have been proposed, called the direct, indirect, and hyperdirect. The original circuitry metric of healthy vs. dysfunctional states was neuronal firing rate ('rate model'), which has been increasingly augmented by resting state functional connectivity derived from MRI techniques (rsfMRI) [18].

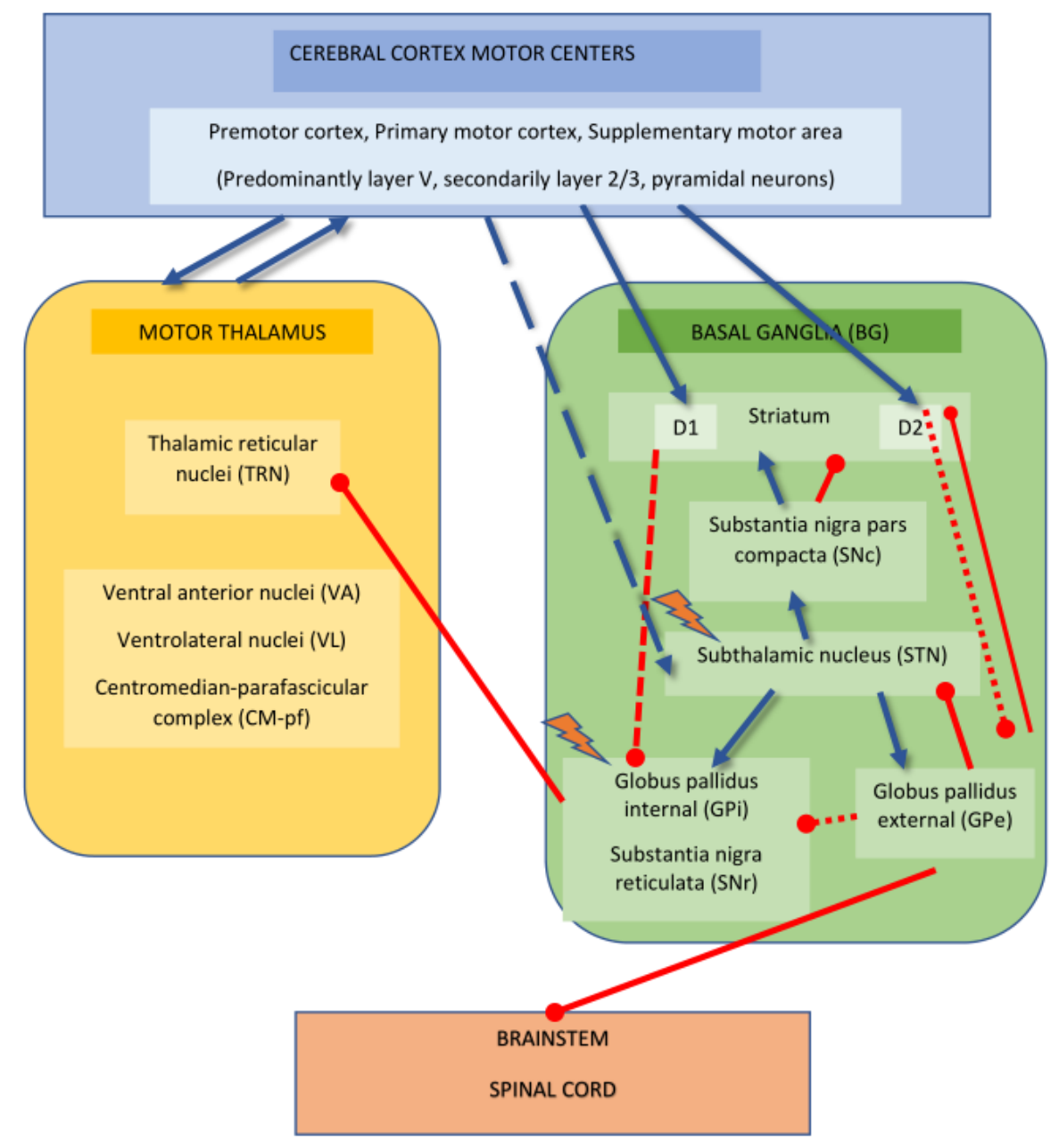

Figure 1 Schematic of the basal ganglia (green), motor thalamus (yellow), motor cortex (blue), and brainstem and spinal cord (brown). There are two input nuclei, the striatum and the subthalamic nucleus (STN), comprised of: 1) Direct pathway: red dotted line from D1 striatal neurons to the globus pallidus internal nucleus (GPi); 2) Indirect pathway: red dashed line from D2 striatal neurons to the globus pallidus external nucleus (GPe); 3) Hyperdirect pathway: blue long-dotted line from cortex to STN. The basal ganglia output is GPi to motor thalamus to motor cortex, or GPe to brainstem to spinal cord. Deep brain stimulation (yellow lightning zigzags) is applied to BG nuclei STN or GPi. 


\subsection{Direct and Indirect Pathways}

Synthesizing evidence from anatomy, physiology, and pharmacology, Albin et al. and DeLong et al. proposed a parallel processing action-selection model incorporating a direct and an indirect pathway comprising positive and negative feedback loops between the BG, thalamus and motor cortex, later empirically supported by Nambu et al. [12, 13, 38]. Functionally, the indirect, polysynaptic pathway from the BG striatum to the GPe and STN is hypothesized to globally inhibit a somatotopically-organized repertoire of motor programs triggered by GPi and SNr. This inhibition continues until the direct, monosynaptic pathway from the striatum inhibits the specific, somatotopic inhibitory group in the GPi/SNr targeting the desired motor program, thereby releasing it from inhibitory control and selecting it for action. The indirect pathway is a deadman's 'brake' signal (i.e. are always on brake) and the direct pathway is a 'go' signal [39]. Or in computer science terms, these circuits are a rapid-response multiplexer for selecting from a repertoire of action sequences. Focal injections of the GABA-A antagonist bicuculline (a chemical lesion) in the striatum results in loss of specificity in BG activity and twitches in specific muscle groups, demonstrating the somatotopic mapping [40].

The targets of the BG output nuclei GPi/STN are the motor regions of the thalamus, which targets the motor cortex, which sends motor program requests to the BG. The BG then processes and relays them to the brain stem and the spinal cord, where they are parsed into an ancient repertoire of motor programs and sent to the relevant muscles via efferent connections from the ventral cord [6$8,13,37,41]$.

The largest component of the BG, the striatum $\left(10^{7}\right.$ neurons), receives excitatory glutamatergic input from the motor cortex (the primary motor cortex, supplementary motor area, and premotor cortex) and is the main input relay center to the rest of the BG [42]. Via its medium spiny projection neurons (MSNs) that comprise $95 \%$ of it, the striatum projects inhibitory dopaminergic input to SNc and VTA [11]. The cortex requests specific motor programs (volitional movement control) via the striatum's fast spiking interneuron excitatory inputs (FSI, $5 \%$ of cells), and the BG regulates the proper selection of each desired program before sending it to brainstem, which processes them and sends them to the spinal cord (automated movement control) [43]. The striatum has two groups of GABAergic MSNs, D1 and D2, characterized by two different dopaminergic inhibitory receptors [8]. Striatal D1 cells project monosynaptically to the excitatory BG output nuclei GPi and SNr, both GABAergic, inhibiting them in the direct pathway, while the D2 cells project polysynaptically to BG output nuclei via inhibition of GPe and STN in the indirect pathway $[7,8,13,44]$. Dopaminergic input from the SNc increases sensitivity to cortical excitatory input to D1 cells and decreases sensitivity of cortical input to D2 cells. Interestingly, MSNs can receive excitatory glutamatergic cortex input on their dendritic heads and inhibitory dopaminergic SNC/VTA input on their dendritic spines, permitting complex modulation [39]. Similarly, dopamine increases FSI activity by concurrently depolarizing the cell via its D5 receptor and reducing GABA input via the D2 receptor, and other neurotransmitters are involved [43]. The striatum's FSIs play an important role in regulating MSN projection activity via recurrent and feedforward inhibition, and MSNs receive inhibition as well from GPe Type A neurons [40]. 


\subsection{Hyperdirect Pathway}

The hyperdirect pathway, running from motor cortex layer $V$ pyramidal neurons to STN, so-called by Nambu et al. since it bypasses the rest of the BG, is hypothesized to be the pathway through which ipsilateral motor actions are 'requested' by the cortex to the BG via excitatory, glutamatergic connections $[11,12,45,46]$. It has also been suggested to suppress inappropriate movement and fine-tune motor circuitry signaling (Vanduffel and Arsenault, 2016) [8, 11]. The hyperdirect pathway has a latency of just 1-3 ms compared with 18-25 ms for the direct pathway, which runs through the GPi/SNr and thalamus to cortex $[18,47]$. While it is not known whether PD MD-related beta band peaks originate exclusively within the BG or require the cortex, DBS PD was hypothesized to affect the cortex via antidromic activation from the STN [48]. The computational model of Anderson et al. predicts that in high frequency PD DBS, if $15 \%+$ of the hyperdirect pathway is affected, antidromic APs suppress synaptic current, which 'lesions' the cortex-STN circuit, preventing generation or propagation of oscillatory activity [49]. However, recent work calls these conclusions into question (see below) [50].

\subsection{Other Relevant Pathways}

While most investigations of the origins of PD movement disorder (MD) focus exclusively on the basal ganglia (BG) (commonly the pallidal-STN loop), other circuitry, notably the thalamus, corticothalamic loop, and the BG-cortico-thalamic loop, may contribute to pathological BG processing and therefore are all open to test by modelling (see Figure 1 in van Albada et al. [8]). Likewise, it should not be ignored that DBS for non-MD symptoms such as pain may be valuable sources for DBS PD for MD model calibration and validation [51].

Modelers may incorporate compensatory mechanisms that reconfigure large-scale motor circuit regulation. Shine et al. found that integration of activity across the medial frontal, lateral parietal, and anterior temporal cortices was inversely correlated with motor symptom severity [52]. Similarly, a recent study pioneering the combination of several empirical techniques, and therefore possibly revealing new phenomena, shows that models may have to include cortical motor areas, reinforcing the significance of the hyperdirect pathway [53]. Ruppert et al.'s multimodal imaging showed reduced rsFC between a dopamine-depleted striatal putamen and cortical motor areas versus healthy state connectivity.

\section{Current Theory of DBS PD Mechanism of Action at the Circuit Level}

Four distinct and conflicting MoA have been proposed for DBS PD at the circuit level [23, 24]:

1. Direct inhibition of neural activity

2. Direct excitation of neural activity

3. Information 'lesion', jamming, or blocking

4. Synaptic filtering

Different MoAs may underly high versus low frequency DBS PD and in either case more than one MoA may be involved [23].

Direct inhibition of net STN or GPi activity was shown to not occur, although suppressing the excitatory sources of aberrant inhibitory interneurons that pathologically are disabled in inhibiting downstream hyperactivity could lead to depressed thalamic activity and akinesia (Figure 2) [23]. 
Direct excitatory stimulation could work similarly, but conversely to direct inhibition, by increasing the activity of inhibitory interneurons to suppress pathological over-active STN or GPi neurons. Either MoA ultimately works by preventing DB-related synchronization or other pathology from propagating throughout the motor control network.


Figure 2 Comparison of healthy (left) vs. dysfunctional PD states (right) due to dopamine depletion in striatum dopaminergic neural groups D1 \& D2. Left: Healthy state with normal numbers of dopaminergic neurons. All connections are represented by lines of the same thickness indicating normal resting state connectivity strength. Right: Parkinson's disease state with dopamine depletion, where line thickness indicates change in connection strength vs. healthy state. Net effect is elevated activity through most of the circuit resulting in pathological synchrony. The difference between the diseased and health states could be measured, e.g. by changes in firing rates in the nuclei or power spectra, as could the degree of restoration of the healthy state via PD DBS. GPe: globus pallidus external. STN: subthalamic nucleus. GPi: globus pallidus internal BSt: brain stem. Thal: thalamus. After Humphrey et al., but with effects of depletion added [37].

A problem with the direct action and lesion hypotheses is that they may imply that normal motor control signalling may be impaired during DBS, yet studies show that sensorimotor signalling occurs in the pallidal centers under DBS to STN or GPi, as well as motor sequence learning and rewardbased reinforcement learning, which is another function of the BG [24]. These issues engender a more refined hypothesis that DBS filters out pathological activity while allowing normal motor control signals to pass.

Thus, the synaptic filtering hypothesis also solves the prima facie paradox of DBS PD stimulating higher activity in STN or GPi yet decreasing oscillatory activity: HF DBS PD over-stimulates cells to 
the extent that synaptic failure occurs, effectively reducing the frequency seen by axons. The resulting lower frequency acts as a filter that attenuates elevated pathological oscillations [54]. A second hypothesis is low-pass thalamic filtering, i.e. the thalamus passes healthy motor signalling (e.g. $\leq \beta$-band $13-35 \mathrm{~Hz}$ ) while HF DBS PD (e.g. at twice the frequency in the circuit) blocks pathological activity $[24,55,56]$.

A recent study by Johnson et al. indicates that the MoA of DBS is orthodromic, as DBS to GPi produced no antidromic activity in the motor cortex, while DBS within STN produced only acute motor cortex activity over a 4-hour period, although therapeutic benefit was maintained as activity waned [50]. Malekmohammadi et al. found that HF DBS (185 Hz, $90 \mu \mathrm{s})$ to GPi reduced low beta band power (13-18 Hz) with no corresponding effect in the motor cortex, while high beta power was suppressed in both GPi and motor cortex [20]. Both studies draw attention away from hypotheses that DBS effects occur via the hyperdirect pathway, while not commenting on the role of the hyperdirect pathway in motor control. This work supports the orthodromic theory of DBS PD that, with SNc dopaminergic neuronal loss, GPe inhibition of STN weakens, producing elevated synchrony, in turn producing PD MD symptoms by blocking normal BG-MC signaling. DBS to dorsolateral STN or GPi reduces BG-thalamo-cortical synchrony and synchrony within the cortical motor areas, as indicated by beta-band power, thus removing the 'information block', restoring normal BG-MC signaling via thalamo-cortical projections [20, 21, 51]. If further studies reinforce this conclusion, it is a major clarification of DBS PD MoA and would help to focus modelers' efforts, beginning with reproducing the results of Johnson et al. and incorporating the required parameters as a calibration of models including the motor cortex.

The varied mechanisms proposed for DBS PD support Ashkan et al.'s call for changing the term 'deep brain stimulation' to 'deep brain modulation' to more accurately characterize DBS' MoAs [23].

We omit investigations into neurotransmitter MoA other than dopamine depletion as they are, as of now, typically incorporated into the overall inhibitory or excitatory connection strength in circuit-level modelling and not as individual channels. Even in active fiber models where there exist, e.g. a dozen different sodium and potassium channels, a 'lumped' approach is taken to capture the net effect of each ion channel type. However, as the science and practice of electroceuticals (neuromodulation replacing the efficacy of drugs), modelling detail at the neurotransmitter level (e.g. glutamate, gamma aminobutyric acid, calcium, serotonin, noradrenaline) may become important. The interested reader is referred to Stefani et al., McIntyre \& Anderson, and Jakobs et al. [56-58].

\section{Effects of Dopamine Depletion in Neuron- and Circuit-Level Models}

From the movement disorder circuit-level theories described above, it is easy to see how, at least prima facie in PD, the loss of the inhibitory, dopaminergic input from SNc would result in dysregulation of the striatum, the largest BG component and dominant relay to the rest of the movement-controlling BG. To reveal specific MoAs, modelers must, however, take a deeper dive into the range of predicted effects [37].

Following the DeLong et al. model of motor program selection and switching in the BG, an early, detailed spiking neuron model by Humphries et al. exemplified predictions of the role of dopamine in healthy and diseases PD states (Figure 2, Table 1 and Table 2) [38, 41]. 
Table 1 Cascade of circuit effects under dopamine depletion in striatum dopaminergic neural groups.

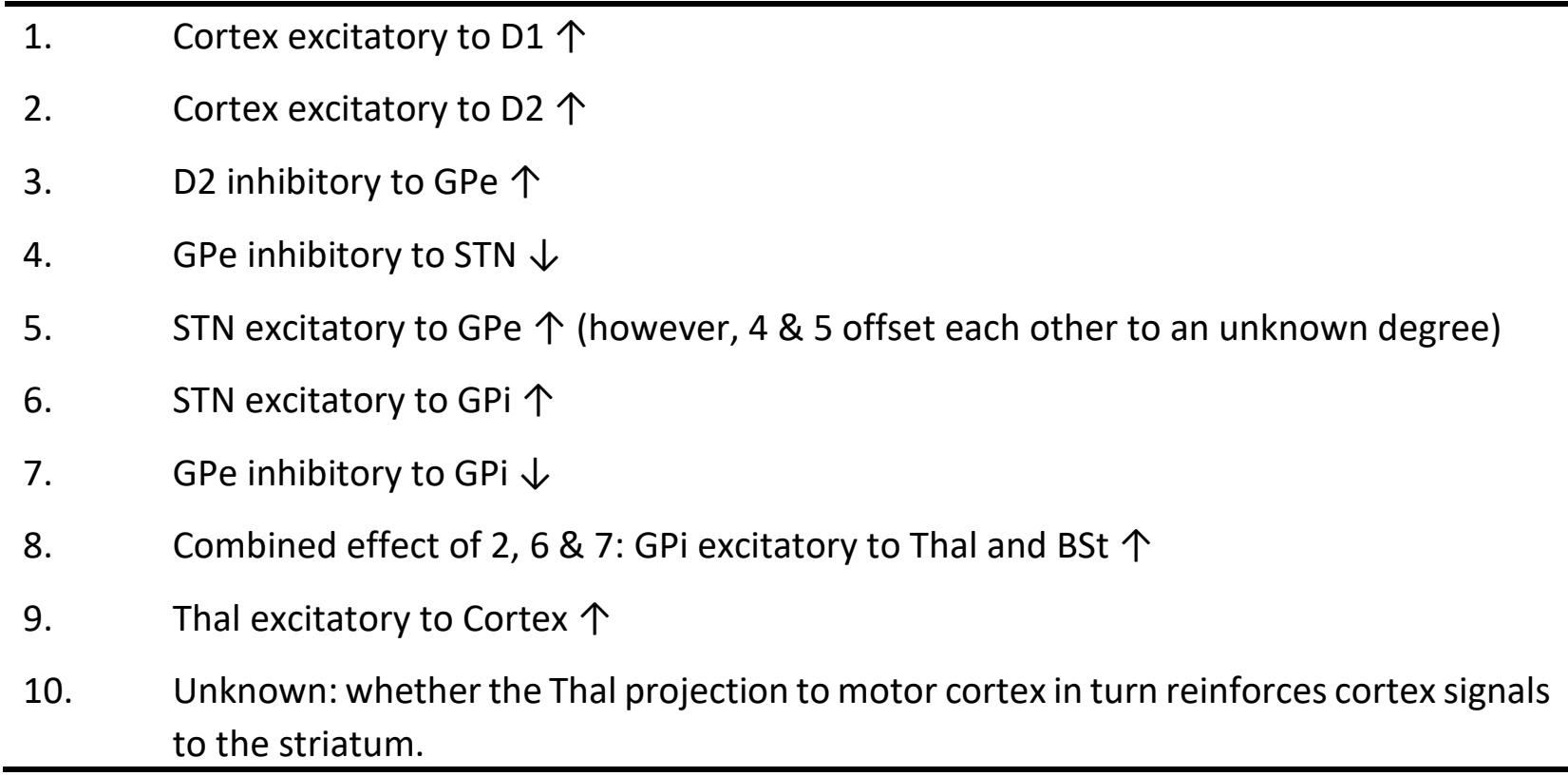

Cortex: motor cortex. D1, D2: two striatum populations of dopaminergic neurons. GPe: globus pallidus external. STN: subthalamic nucleus. GPi: globus pallidus internal BSt: brain stem. Thal: thalamus.

Table 2 Model and empirical elucidation of the role of dopamine in healthy and depleted states at the neuron and neural circuit levels.

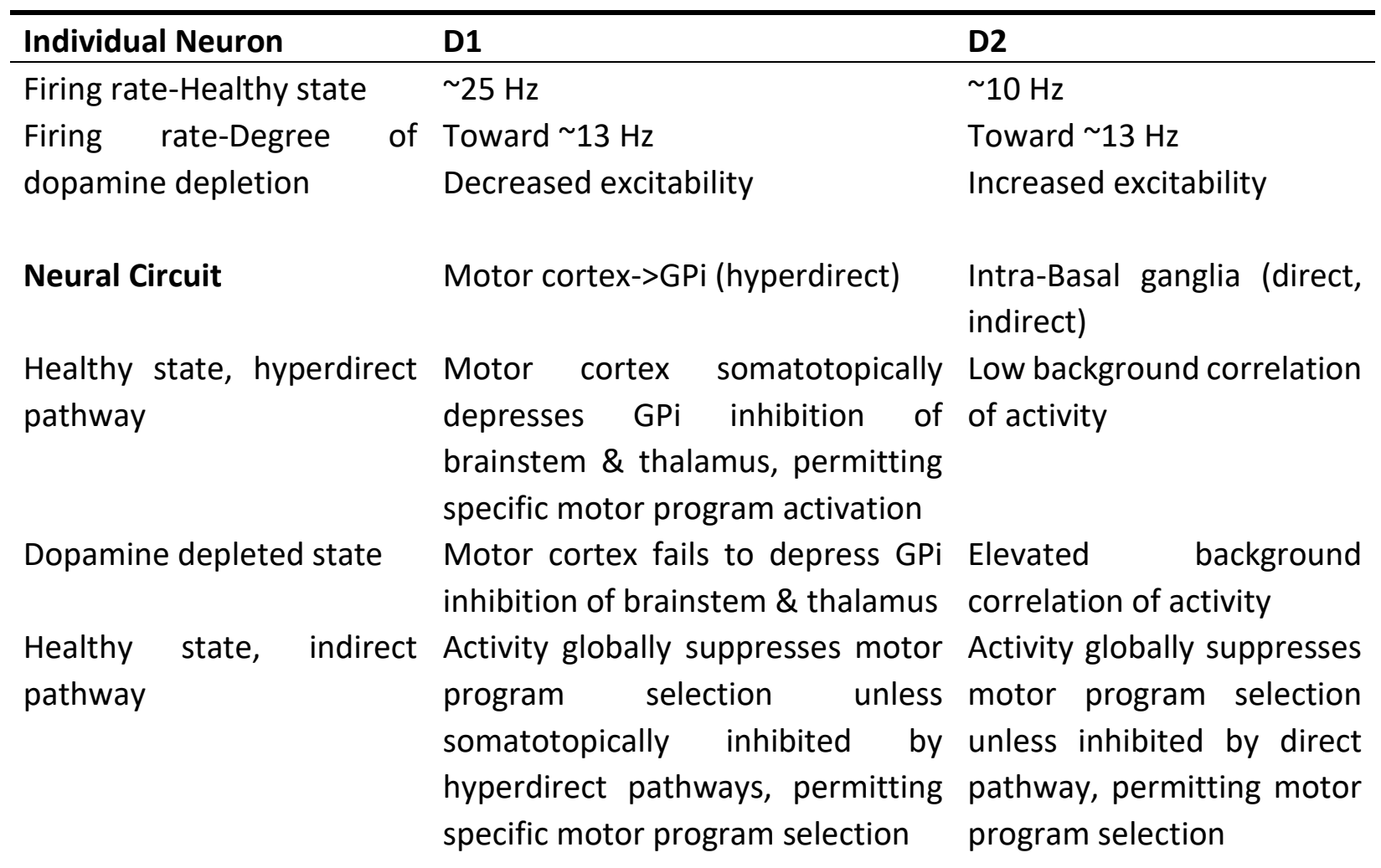


Dopamine-depleted state Elevated inhibitory activity reduces Elevated inhibitory activity inhibition by hyperdirect pathway, reduces inhibition by direct inhibiting movement pathway, inhibiting movement

At the circuit level, loss of dopamine results in elevated indirect pathway activity via the STN and weakened hyperdirect pathway activity. Together these results are theorized to prevent normal suppression of the indirect pathway by the direct and hyperdirect pathways, and thereby hinder proper movement program selection and modulation [46]. We detail the cascade of circuit effects from dopamine depletion in D1 and D2 in Figure 2 and Table 1. Figure 2 is representative of a simple schematic to illustrate an experiment to be modelled, where metrics such as firing rates in the nuclei or power spectra can be used to measure the difference between the healthy and diseased states and the degree of healthy state restoration via neuromodulation. It can be seen that the net effect of dopamine depletion at the circuit level is elevated excitatory activity. From this we hypothesize that a background signal-to-noise ratio that is exceeded by motor-program selection commands in the healthy state is replaced by a noisier state characterized by pathological synchrony, and that DBS PD interrupts the synchrony, attenuating noise back toward healthy state levels.

Further calibration data for a dopamine-depleted circuit is provided in Table 2.

\section{Basic Requirements for PDS DB Modelling Studies}

Augmenting a similar list from Holt \& Nethoff [31], a conceptually complete DBS PD model must include the following:

1. Biomarkers to measure calibration and validation of healthy and diseased states

2. Well-defined descriptions of healthy and diseased states at appropriate systems levels

3. One or more neuromodulation protocols hypothesized to ameliorate a given pathology

4. Hypotheses of how each neuromodulation protocol affects neural activity and restores the healthy state from the diseased state

5. Possible in silico, in vitro, or in vivo experiments to verify or falsify the hypotheses

\section{Model Calibration and Validation}

An extensive set of criteria that can be used for calibration and validation of models is given in the Supplement. Any number of the given criteria can be used to calibrate a model, and then running the model to see if it manifests any of the remaining criteria can constitute a validation. In general, biological models tend to be under-validated. Model robustness benefits from incorporating calibration and validation data that are in some sense orthogonal to each other.

\section{Types of DBS PD Computational and Numerical Models}

Neural circuitry computational models typically involve a way to represent processing of inputs to neurons from other neurons and output from neurons to other neurons in a software program, some having more detail in such processing, some less, some more biologically inspired, some lessso. Modelling can capture detail at several biological systems levels, which affect methods of calibration, validation, as well as model complexity. 


\subsection{Top-Down Organizing Principles}

Holt \& Netoff surveyed computational and numerical models of epilepsy as well as DBS PD and classify DBS PD into two categories with different goals: (1) abstract models that replicate behaviours but are not clinically predictive, and (2) models that are clinically predictive, incorporating and solving for patient-specific parameters [31, 59]. These are idealized categories, and most modelling is a mix of the two categories and one way or another embody a 'bench-tobedside' paradigm. A purely theoretical model typically must still incorporate clinical parameters to be useful and a purely clinical model whose aim is to improve clinical parameters typically still needs to be distilled down to an abstract model.

In the last two decades the top-down method of imaging correlated functional brain activity to create a high-level resting state functional connectivity connectome ( $\mathrm{rsFC}$ ) of the healthy and diseased states has become increasingly effective in analyzing many neurological disorders [60-62]. In rsFC the biomarker dimensions provide a way to describe healthy and diseased states and a 'distance' between diseased and healthy states. Testable hypotheses can be framed in terms of the degree to which neuromodulation restores the healthy state along one or more rsFC dimensions. The rsFC paradigm is likely to provide the over-arching framework into which DBS PD MoA and efficacious parameters will be organized in the future.

\subsection{Trade-offs in Neural Circuit Model Granularity Categories}

\subsubsection{Active Fiber Models}

Active fiber models containing multiple ion channels with voltage-modulated time factors that underlie conductances are often needed to capture specific neuron characteristics such as synaptic transmission failure, threshold and propagation blocking, response to high frequency stimulation (e.g. 1-30 KHz), action potential shape such as the after-potential or short- and long-term threshold increase or decrease (threshold 'accommodation') [54, 63-75]. More generally, highly specialized numerical and computational models may be developed using a minimal, carefully triaged set of underlying assumption to capture specific neural phenomena [76].

\subsubsection{Mean Field, Driving Force, and Volume of Tissue Activated Models}

Gunalan et al. 2018 sought to compare the accuracy of three categories of DBS PD models: 1) field-cable, which includes active fiber models coupled with FEM of the electric field, and which is most computationally complex and time-consuming, but also most accurate, 2) driving force, which is in the middle of the complexity spectrum, and 3) volume of tissue activated (VTA), which is least complex, and as they found, least accurate [77].

Neural mean field theory is a high-level, computationally efficient mathematical framework typically used for modelling large neural circuits. The term 'mean field' derives from the use of spatial and temporal averages in discrete, coarse-grained volumes and time intervals to describe distances between neural populations at spatio-temporal position and numbers of APs in a given time, e.g. firing rate $Q_{a}$ (where $a$ is for average) at position $r$ and time $t: Q_{a}(r, t)$ [78]. The dramatic reduction in degrees of freedom results in computational efficiency and transparency [79]. Rise and fall sides of the AP can be described by exponential, sigmoid, or step functions. A potential 
shortcoming is the use of just two neuron types, excitatory or inhibitory. Mean field theory's framework matches that of rSFMRI and describing the difference between healthy, diseased, and neuromodulated states [15]. We see significant advantages in marrying neural field models with more detailed bottom-up approaches that incorporate, e.g., different neural types as do Kumaravelu et al. [29, 80].

VTA models typically incorporate several simplifying assumptions: 1) isotropic tissue conductivity, 2) straight axons oriented perpendicular to the applied electric field, 3) finite volumes of tissue proximate to the electrode, all for parameters of fiber diameter, specific electrode array activation, pulse width, and amplitude [77, 81, 82]. Note that assumptions $1 \& 2$ can be loosened for greater accuracy by using anisotropic conductivities in multiple tissues and axons of any orientation to the field. Also, while VTA commonly requires an additional assumption that AP frequency match stimulation frequency ('1:1 response'), we suspect this requirement is spurious and misleading, based on other neuromodulation paradigms (e.g. spinal cord stimulation and vagus nerve stimulation), in which efficacy can be achieved without 1:1 response.

The intermediate complexity category, driving force models, significantly reduces complexity of field-cable models by using a metric for activating an axonal AP [77, 83-87]. The inherent limitations of the simplified approach can be ameliorated by incorporating distance-weighted potential contributions to the excitation of a given node of Ranvier ( 2 adjacent nodes in Rattay's 'activating function' and an unlimited number in Warman's 'driving function'), or calibration to exogenous passive or active models [85, 88-91]. The accuracy gain of $20 \%$ under certain circumstances may not be necessary for most model goals or as a first pass model.

Ultimately, DBS PD model types are strategies to efficiently bridge several biological systems levels (multi-scale modelling) by incorporating no more detail from each than is needed to capture the phenomena of interest, thus, models must be judged in the context of their goals and no method is 'best' [29]. Our categorization of the models reviewed here is given below.

Table 3 Reviewed models categorized according to computational complexity in bridging biological systems levels inherent in representing DBS PD (most complex, left, to least complex, right).

\begin{tabular}{|c|c|c|c|}
\hline $\begin{array}{l}\text { Active Fiber } \\
\text { (aka 'Field-Cable') }\end{array}$ & Mean Field & Spiking Network & $\begin{array}{l}\text { Volume of Tissue } \\
\text { Activated (VTA) }\end{array}$ \\
\hline Gunalan \& Mclntyre & van Albada et al. & Lindahl et al. & Yousif Purswani et al. \\
\hline Lempka Howell McIntyre & Muller \& Robinson & Izhikevich & \\
\hline Arle et al. (hybrid) & & Thibeault et al. & \\
\hline Kumaravelu et al. & & Arle et al. (hybrid) & \\
\hline Kang \& Lowery & & & \\
\hline
\end{tabular}

\section{Representative Survey of DBS PD Numerical Models}

\section{1 van Albada et al.}

A notable exemplar of modelling DBS PD is the paradigm of van Albada et al., which captured the direct, indirect, and hyperdirect pathways, 5 types of progressive dopamine loss, and sought to 
identify differences between healthy and PD states [8, 92]. Their study is also an early example of using functional connectivity to distinguish the healthy and diseased states and map the model to known anatomical regions. The work exemplifies a balance of the qualities of 1) exemplary knowledge of PD and literature review for hypotheses to test and calibrations, 2) sophisticated numerical methods, and 3) a rich set of phenomena captured at the appropriate systems level, in this case using mean field theory for efficient simplification of a complicated system. Most modelling studies are under-weighted in one or two of those categories. Concomitantly, the downside of their framework is that it is on the complex side of the modelling spectrum and involves sophisticated mathematics, which limits access and reproduction of results to a subset of modelers. A reasonable use of the van Albada et al. work would be for modelers to create a simpler way to capture a salient subset of their paradigm, e.g. progressive dopamine loss, and compare results with those of the more complex model.

\subsection{Gunalan \& Mclntyre}

Gunalan \& Mclntyre sought to model DBS PD recruitment of the hyperdirect pathway, as suggested by Li et al., who noted that DBS targeting STN will generate axonal APs in both directions along the axon, i.e. orthodromically to STN's BG target, GPi, or antidromically back to the cortex, and cited supporting evidence of antidromic effects [48, 93] (but see conflicting evidence in [50]). The Gunalyn-McIntyre model is quite sophisticated in a number of respects, such as the detailed MRI data underlying its design and calibration, the use of an active fiber nerve model that is, in itself, quite sophisticated [64], and the design of the neural circuitry. Notably, Gunalan \& Mclntyre conclude that the evoked potential (EP) $\sim 3 \mathrm{~ms}$ EP1 and $\sim 5 \mathrm{~ms}$ EP2 signals are correlated with antidromic activation of the hyperdirect pathway, and specifically with the 10 and $5.7 \mu \mathrm{m}$ fibers in their model. While prima facie this seems significant, their model only included the three diameter axons, and these seem too large for the cortico-BG circuitry, which tracing studies specifically of the hyperdirect pathway show to be $2-3 \mu \mathrm{m}$ in diameter (and the article describes them as 'large caliber' in the context of cortical axons) [46]. A further criticism is that their nerve model was calibrated to certain motor fiber behaviour such as threshold and AP shape, and the thresholds may be unreliable below the typical large diameter of motor axons. While these observations suggest possible flaws in the Gunalyn-McIntyre methodology, the model is sophisticated, original, and worth re-building with accurate calibration parameters or re-using its components in a different, and perhaps simpler, model.

\subsection{Lempka Howell McIntyre}

Integrated circuit pulse generators (IPG) are used to produce waveform shapes intended by DBS protocol designers, but what waveforms are actually produced? An influential paper by Geddes illuminated this question from the standpoint of voltage-driven vs. current-driven IPGs, showing that current-driven IPGs preserve rectangular waves with high-fidelity while voltage-driven IPGs do not, and consequently manufacturers converted some protocols from voltage-driven to currentdriven [94]. Lempka et al. analysed actual IPG fidelity specifically for protocols and waveforms used in DBS, simulating IPG circuits with simplified electronically-equivalent model circuits calibrated to bench IPG output recordings [95]. They found significant differences for long bipolar pulses, and average errors were $\sim 3-24 \%$ for constant-voltage stimulation and $\sim 2-11 \%$ for constant-current 
stimulation. Lempka et al. note the importance of using accurate waveforms in computational modelling DBS to predict efficacy, e.g. via metrics such as VTA. Another factor motivating manufacturers to adopt constant-current stimulation was its ability to preserve the strength and shape of the electric field at the target dorsal column as electrically-resistive scar tissue forms under the electrodes (fibrosis) [96]. Uneven fibrosis not only can weaken the field, but counter-intuitively, can strengthen the field and distort the intended distribution, such as when scar forms under a 'guarding anode' that is restricting the penetration of cathodic pulses [90]. These theoretical predictions were borne out in a study showing greater patient satisfaction and pain relief with constant-current vs. constant-voltage stimulation [97].

\subsection{Lindahl et al.}

Noting that previous computational and animal models had failed to uncover the global effects of numerous local effects in the BG of dopamine loss, Lindahl et al. attempted to model the BG in a larger, coarse-grained network context, calibrated with known local dopamine loss effects [40].

While coarse-grained (7 neural groups), Lindahl et al.'s spiking network model is nonetheless quite complex (80,000 neurons, 11 neuron parameters, 5 neuron types, $\sim 100$ synaptic parameters, $\sim 10^{6}$ connections, and various other parameters) and reproducing their results, re-purposing, or modifying the model would be a significant undertaking. Thus, the paper is a rich source of parameter values and calibrations for modelers.

\subsection{Izhikevich}

An insightful description and comparison of spiking neuron (aka 'integrate-and-fire') models, their benefits, computational efficiency, usage in network models, and tuning phenomenologically (i.e. to firing characteristics vs. to empirical neuron parameter values) is given by Izhikevich [98]. These models derive from the general theory of nonlinear dynamical systems, especially bifurcation theory, the essence of which is described. The term 'hybrid' refers to the two phases of simulation implemented with a piecewise algorithm: 1) computationally detailed, continuous dynamics of the prelude to neuron threshold and the upstroke of spike generation, and 2) computationally simple, discontinuous and linear 'hard reset' to initial conditions after a spike peak voltage defined by the modeler. A clever hack is used to transition time-stepping from a relatively coarse-grained step (typically $1 \mathrm{~ms}$ ) leading up to a spike and a finer-grained timestep in the vicinity of the spike to capture the rapid spike dynamics. However, a trade-off occurs between the computational efficiency of using large time steps and numerical instability triggered by large synaptic currents. Instead of using computationally-intensive numerical methods (e.g. backward or 'implicit' Euler, higher-order Runge-Kutta) to solve the differential equation for membrane potential, Izhikevich sacrifices accuracy in the down-spike shape by using a linear equation.

\subsection{Thibeault et al.}

A considerably simpler and more manageable spiking neural network model to compare with Lindahl et al. is that of Thibeault \& Srinivasa, containing 4 neuron types and groups, 5 neuron parameters, 5 synaptic parameters, and 12 sets of connections modulated by just 2 parameters [99]. Notably, the Thibeault \& Srinivasa study is an exemplar of reducing model complexity in their critical 
selection of four previous hybrid models of the BG and their simplification of the models. They provide succinct overviews of a selection of earlier models and the hybrid spiking model comparing their different topologies. They analyzed the action-selection theory of BG and the PD diseased state but fall short of being able to offer improvements on DBS protocols.

While not using the term 'functional connectivity,' Thibeault \& Srinivas, in their usage of correlated firing rates in healthy vs. PD states, implicitly align with the functional connectivity paradigm. Although the model is considerably simpler than others (e.g. Lindahl et al.), Thibeault \& Srinivas nonetheless largely succeeded in calibrating their BG neural firing patterns to empirical data, comparing their results to those of earlier models, e.g. Humphries et al. [41]. They argue that some BG firing patterns require enough complexity to model individual neurons as well as network- or population-level effects. This issue in general will remain central to ongoing discussions of realistic and efficient connectome modelling [29]. Thibeault \& Srinivas' theme is that the use of simpler models may reveal mechanisms of action that are difficult to parse out in more complex models. Besides their Izhikevich hybrid neuron, they point to the adaptive-exponential-integrate-and-fire model [100], and secondarily, rate- and population-based models. They stress the unreliability of using hybrid neurons in small networks vs. large-scale ones and recommend biologically more realistic conductance-based models for simulating individual neurons or small networks.

\subsection{Arle et al.}

Along those lines, the hybrid UNCUS universal network simulator captures both individual neuron and network-level phenomena efficiently [101]. The model can simulate individual neurons with any characteristic current-voltage curve and networks containing them, including ion channel conductances, threshold accommodation, and connectivity strengths set globally, or locally along the dendritic tree, as well as in the density of source-target connections. A lumped nonlinear conductance parameter permits user-defined cell model expansion by using the parameter to tune to a given neuron's characteristics, store the neuron in the repertoire of model neurons, and call it as needed at the population level. Notably, incorporating computationally efficient features from MacGregor, Rall, and McNeal, their framework scales efficiently to model small-to-medium sized networks such as DBS PD $[101,102]$ or as large as the human spinal cord containing $10^{6}$ neurons $[87,103-105]$. In contrast to models on the more complex side of the spectrum such as van Albada [8], the Arle et al. model is accessible and can be programmed by anyone familiar with numerical solutions of differential equations. The principal calibration for modelling PD DBS was background BG firing rates in humans and the key result was DBS 'resetting' firing rate variance in PD and predicting efficacy at stimulation frequencies $>130 \mathrm{~Hz}$ in agreement with clinical reports.

\subsection{Kumaravelu et al.}

Kumaravelu et al. offer specific analyses of how DBS works and what protocols are optimal, according to their model [80]. Noting that earlier computational studies replicated primate animal models of PD but were not calibrated to the 6-OHDA rat PD model, they used that calibration and wanted to find what this particular model showed about DBS PD to the STN. Interestingly, Kumaravelu et al. used 7 complex neuron models incorporating up to 11 different ion channel dynamics, and compensated for the lower-level complexity at the network level by using just 10 neurons per nucleus-striatum, STN, GPe, GPi (which in rat is homologous entopeduncular nucleus, 
EP), thalamus and cortex (containing regular- and fast-spiking neurons). Thus, their emphasis is 180 degrees opposite to rate- or population-based models, and their emphasis on where complexity is needed to capture the key DBS PD phenomena strikingly different than the hybrid spiking models. Despite the bottom-up complexity, the calibration to the diseased state is simple and transparent, involving just 3 neuron parameters capturing 3 effects of striatal dopamine loss: 1) reducing M-type potassium channel conductance, 2) reducing cortico-striatal conductance, and 3) increasing GPe synaptic conductance. The model of Kumaravelu et al., calibrated uniquely to the 6-OHDA lesioned rat model, showed, similar to findings in Arle et al. [102], that $130 \mathrm{~Hz}$ DBS PD disrupted a pathological synchrony in STN, GPi, and GPe nuclei. The notable feature of their study is its focus on a specific model and developing a model that is complex at the lower biological systems level offset by minimal complexity at the immediately higher systems level.

\subsection{Muller et al.}

The Muller et al. study is an exemplary, transparent use of mean field modelling to capture largescale network behavior where the underlying methodology is explicated in detail. They compare the pathological power spectra and synchrony changes resulting from DBS PD to the STN and GPi. The stated goal is to provide a framework to systematically test the effectiveness of different DBS protocols that has been validated against empirical and clinical findings. The focus on steady state firing rate perturbations around a mean value demonstrates the importance of tying mean field to those of rsfMRI.

\subsection{Kang \& Lowery}

Kang \& Lowery hypothesized that DBS PD disrupted pathological synchrony using beta band power as the metric [106] and sought to distinguish orthodromic effects on the STN from antidromic effects on the cortex, and examine the combined effects [106, 107]. They present the empirical evidence from human and rat studies for combined effects, and contrast those with empirical and theoretical arguments that antidromic effects may be negligible or minor. Analyzing and separating out orthodromic and antidromic effects in DBS PD via simulating physical or chemical lesions, and sweeping across parameter ranges that are simply impossible outside of numerical methods, are good examples of what can be done relatively quickly and inexpensively with a computational model vs. in vivo studies [107].

Kang \& Lowery employed numerous simplifications: three neural centers (cortex, GPe, and STN), a point electrode source, homogeneous conductivity, $\sim 100$ hundred neurons/nucleus,random vs. topographic, connectivity, a point source electric field, and a volume conductor model [108]. At the neural level, however, they attempted to capture more detailed effects drawing on different model neurons from other labs for the different nuclei [106].

At the cortical neural level, the model followed Rattay [85] and was detailed anatomically, modelling excitable elements of the neural cell body, axon initial segment (AIS), main axon and axon collateral, while omitting the dendritic level, which may be important for the timing of AP reception (cf. Arle et al. Figure 2, Yousif et al., and Rall [101, 105, 109]). STN and GPe neurons derived from previous models developed specifically for those nuclei. The specialized STN neuron followed the conductance-based model of Otsuka et al., who calibrated to data on how STN is regulated by 'plateau potentials' manifesting during hyperpolarization of particular STN neurons [110]. The STN 
neuron followed the detailed, specific model of Rubin \& Terman [111]. In these cases a simplified model capturing the essential STN neuron current-voltage behaviour would likely suffice, whereas in their previous study STN neuron detail was necessary to capture the effects of dopamine depletion [106]. Last, the computationally efficient Ishikevich spiking model was used for interneurons (see above description [98]).

Kang \& Lowery's calibrations was top-down and notably simple. They initiated beta-band oscillations in the cortex, adjusted cortico-striatal synchrony via synaptic gain adjustment (a simple scaling factor) from cortex to STN, between self-inhibitory GPe neurons, and mutually between STN and GPe until beta synchrony manifested in the STN and GPe [107]. They then used computational 'lesions' to isolate the antidromic and orthodromic pathways under DBS and examine the resulting effects in each circuit separately and with both activated.

Kang \& Lowery's key results are: 1) Orthodromic DBS entrains STN neurons to the stimulus frequency and suppresses pathological beta activity throughout the network; 2) Antidromic DBS achieves the same effect via a different mechanism, to wit, disrupting cortical activity that otherwise (per their calibration) causes or reinforces beta activity in the BG (STN and GPe), and 3) The effects are synergistic. The antidromic result predicts that the cortex is a primary target of DBS PD as well as STN, providing an alternative hypothesis to activation of STN alone and may illuminate identification of DBS side effects via axons of passage (cf. [25, 26, 93, 112-114]). HF DBS (60 $\mu$ s pulse width @ $130 \mathrm{~Hz}$ ) is predicted to reduce STN beta-band power via disruption in both directions and is roughly proportional to frequency between 80 and $130 \mathrm{~Hz}$, while LF DBS would reinforce it via resonance effects. (Note that clinically, DBS PD frequency $>130 \mathrm{~Hz}$ is more effective than lower frequencies and is always used except to treat dystonia.) DBS at $20 \mathrm{~Hz}$ had no effect in either direction.

\subsection{Yousif Purswani et al.}

A thesis of the study by Yousif et al. (2010) of DBS PD to STN was that a detailed model of neural anatomy including the dendritic tree, soma, and axon would reveal significant phenomena missed by previous studies [115]. Following Arle et al. [101, 102], they coupled a finite element model to predict the electric potential distribution in space and time to their neural model. Notably, they compared the DBS metric, VTA, using their neural anatomical model to the typical axon models. Not unexpectedly, to directly fire APs by stimulating neuron cell bodies required over 10x the stimulus amplitude as did axons, but they also found that neurons are stimulated in high current density regions near the electrodes perhaps in sufficient numbers to affect DBS. Yousif et al. found significant variation in VTA across different DBS frequencies, and noted the importance of capturing the effects of DBS frequency as well as the resulting frequency of APs in targets such as the STN. Their statement that frequencies below $100 \mathrm{~Hz}$ worsen symptoms has been proven simplistic by subsequent studies such as those described in the meta-study by Su et al., which found differential effects in the HF and LF DBS ranges [10].

\section{Closed-Loop, Adaptive DBS (aDBS)}

We give an overview of the relatively new goal for DBS PD, 'closed-loop' technology, because it may supersede the current non-closed loop paradigm and modelling will play an important part in its development. Closed-loop refers to a technology wherein recordings of effects produced by a 
neuromodulation stimulus are used as feedback to modify the stimulus in real-time ('adaptive' or ' $a D B S^{\prime}$ ). Besides the advantage of superior protocols tuned to optimize efficacy for different tremor types, a significant potential advantage is greater energy efficiency due to lower duty cycle (ON time to ameliorate MDs), and thus less frequent invasive surgical battery changes for PD patients [22]. Wang et al. make a strong case that 1) MoA understanding is necessary to understand neuromodulation side effect etiology, 2) mechanistic models are necessary to uncovering MoA, and 3) closed-loop protocols developed via the models are necessary to reduce side effects [116].

Closed-loop DBS PD is in an early stage. The closed-loop concept floated for DBS PD is to identify signatures for the several types of PD MDs- 'biomarkers' or 'signatures', such as changes in LFPs in the STN or GPi-and then select from a menu of efficacious DBS protocols a response to each signature. However, neither has a reliable signature been identified for any DBS MD, nor has a specific protocol addressing a type of DBS MD yet been developed [22,117]. Modelling may help overcome these two critical and necessary hurdles. Developing protocol responses to specific MD signatures will lead to reducing side effects such as psychiatric symptoms and speech interference $[118,119]$. Along with improving the accuracy of MD signatures and developing efficacious responses, a third hurdle is to identify the signatures rapidly enough that aDBS algorithms can respond in real-time [118]. However, segmented leads may allow programming to eliminate most side effects without the need of a closed-loop component.

Biometrics based on beta-band power are being replaced by more precise metrics [120] and single signature components, e.g. beta-band power or high-frequency oscillations, have proven insufficient; detecting multiple signature features increases accuracy [121-123]. Hirschmann et al. found that training the machine learning algorithms on a single patient was more accurate than across patients, indicating signatures differ significantly between patients [121]. This finding further strengthens the need to use machine learning in individual aDBS devices and for modelers to encompass the range of a given signature in patient populations. Thus, modelers will need to simulate machine learning to keep up with aDBS technology.

Advances in the application of machine learning techniques may be the route to identifying signatures for tremor types, to which modelling techniques can be applied to develop the effective protocols and open the door to aDBS. The first human clinical trial comparing conventional DBS with aDBS has been planned [120]. The study acknowledges the limitations of beta band power as a biomarker for tremor and will focus on its correlation in STN and GPi with bradykinesia and rigidity, will compare STN versus GPi targeting, test the hypothesis that the intended low duty cycle of aDBS will reduce dysarthria (impaired speech) due to over-stimulation in conventional DBS, and test for side effects in executive function.

\section{Conclusion}

Computational modelling of DBS PD has extensively explored the main phenomena of interest in PD etiology, such as dopamine depletion and dysfunction of the direct, indirect, and hyper-direct signaling pathways. We have reviewed a representative sample of neural simulation methods that have been developed by modelers. While the work done to date has fallen short of decisive unraveling of PD etiology and DBS MoA, it provides a rich paradigm for selecting tools with which to target specific PD phenomena and DBS effects, suggest hypotheses to test, and incorporate refined empirical parameters. At a meta level, computational modelling of DBS PD is an example of 
the general need for modelers, theoreticians, and empiricists to couple their work more tightly. Empirical results should be rapidly incorporated into the models and model predictions should be more rapidly tested empirically [30].

Ultimately, the measure of model utility is the ability to predict therapeutic outcomes and improve protocols and devices. Modelers must judiciously weigh the level of model detail needed to capture diseased vs. healthy states, the etiology of the diseased state, and the restoration of the healthy state due to the applied therapy, vs. limitations, such as transparency and computational efficiency $[29,99]$. Looking to the future of individualized closed-loop PD DBS, portable, energyefficient hardware containing a model of the patient is unlikely to be feasible unless the model is simple enough to run computationally, to understand, and to validate that it runs correctly [99, 124].

\section{Acknowledgments}

We appreciate the help of Dr. Amit Agrawal of Gandhi Medical College in preparing an early draft.

\section{Additional Materials}

The following additional materials are uploaded at the page of this paper.

1. Table S1: General Parkinson's disease calibrations and validations (generally non-power spectrum concepts).

2. Table S2: Firing rates in the basal ganglia, thalamus, and motor cortex for calibration of numerical PD models.

3. Table S3: Signal latencies

4. Table S4: Power spectra calibrations and validations.

5. Table S5: DBS Calibrations.

6. Sample hypotheses for modelers to test.

7. Supplement S7: Signaling Pathways of Parkinson's Disease.

\section{Author Contributions}

KWC researched the literature, assisted by SP, and KWC and JEA wrote the manuscript, of which all authors approved.

\section{Funding}

Funding was generously provided by the Sydney Family Foundation.

\section{Competing Interests}

The authors have declared that no competing interests exist.

\section{References}

1. Rajagopalan SS, Miller AM, de Hemptinne C, San Luciano M, Ostrem JL, Starr PA. Washout of chronic therapeutic deep brain stimulation increases cortical phase-amplitude coupling. Parkinsonism Relat Disord. 2019; 66: 269-271. 
2. Wang DD, de Hemptinne C, Miocinovic S, Ostrem JL, Galifianakis NB, San Luciano M, et al. Pallidal deep-brain stimulation disrupts pallidal beta oscillations and coherence with primary motor cortex in Parkinson's disease. J Neurosci. 2018; 38: 4556-4568.

3. Montgomery EB. Deep brain stimulation programming: Mechanisms, principles, and practice. 2nd ed. New York: Oxford University Press; 2017.

4. Arle JE, Shils JL. Essential neuromodulation. 1st ed. Amsterdam: Elsevier/Academic Press; 2011.

5. Arle JE, Shils JL. Innovative neuromodulation. London: Academic Press; 2017.

6. Vanduffel W, Arsenault JT. Should I stay or should I go? Neuron. 2016; 91: 207-210.

7. Albin RL, Young AB, Penney JB. The functional anatomy of basal ganglia disorders. Trends Neurosci. 1989; 12: 366-375.

8. van Albada SJ, Gray RT, Drysdale PM, Robinson PA. Mean-field modeling of the basal gangliathalamocortical system. II: Dynamics of Parkinsonian oscillations. J Theor Biol. 2009; 257: 664688.

9. Chan CS, Surmeier DJ, Yung WH. Striatal information signaling and integration in globus pallidus: Timing matters. Neurosignals. 2005; 14: 281-289.

10. Su D, Chen H, Hu W, Liu Y, Wang Z, Wang X, et al. Frequency-dependent effects of subthalamic deep brain stimulation on motor symptoms in Parkinson's disease: A meta-analysis of controlled trials. Sci Rep. 2018; 8: 14456.

11. Nambu A, Tokuno H, Hamada I, Kita H, Imanishi M, Akazawa T, et al. Excitatory cortical inputs to pallidal neurons via the subthalamic nucleus in the monkey. J Neurophysiol. 2000; 84: 289-300.

12. Nambu A, Tokuno H, Takada M. Functional significance of the cortico-subthalamo-pallidal 'hyperdirect'pathway. Neurosci Res. 2002; 43: 111-117.

13. Alexander GE, Crutcher MD. Functional architecture of basal ganglia circuits: Neural substrates of parallel processing. Trends Neurosci. 1990; 13: 266-271.

14. Parkinson's Foundation. Statistics [Internet]. Miami, FL: Parkinson's Foundation; 2020. Available from: https://www.parkinson.org/Understanding-Parkinsons/Statistics.

15. Gilson M, Kouvaris NE, Deco G, Mangin JF, Poupon C, Lefranc S, et al. Network analysis of wholebrain fMRI dynamics: A new framework based on dynamic communicability. Neurolmage. 2019; 201: 116007.

16. Gu JW. Subthalamic nucleus deep brain stimulation for Parkinson's disease. In: The neuromodulation casebook. San Diego: Elsevier Science \& Technology; 2020.

17. Benabid AL, Chabardes S, Torres N, Piallat B, Krack P, Fraix V, et al. Functional neurosurgery for movement disorders: A historical perspective. Prog Brain Res. 2009; 175: 379-391.

18. Udupa K, Chen R. The mechanisms of action of deep brain stimulation and ideas for the future development. Prog Neurobiol. 2015; 133: 27-49.

19. Benabid A, Wallace B, Mitrofanis J, Xia R, Piallat B, Chabardes S, et al. A putative generalized model of the effects and mechanism of action of high frequency electrical stimulation of the central nervous system. Acta Neurol Belg. 2005; 105: 149-157.

20. Malekmohammadi M, Shahriari Y, AuYong N, O'Keeffe A, Bordelon Y, Hu X, et al. Pallidal stimulation in Parkinson disease differentially modulates local and network $\beta$ activity. J Neural Eng. 2018; 15: 056016.

21. Weiss D, Klotz R, Govindan RB, Scholten M, Naros G, Ramos-Murguialday A, et al. Subthalamic stimulation modulates cortical motor network activity and synchronization in Parkinson's disease. Brain. 2015; 138: 679-693. 
22. Muthuraman $M$, Bange $M$, Groppa S. Advanced technologies for detecting tremor in Parkinson's disease. Clin Neurophysiol. 2020; 131: 241-242.

23. Ashkan K, Rogers $P$, Bergman H, Ughratdar I. Insights into the mechanisms of deep brain stimulation. Nat Rev Neurol. 2017; 13: 548-554.

24. Lozano AM, Lipsman N, Bergman H, Brown P, Chabardes S, Chang JW, et al. Deep brain stimulation: Current challenges and future directions. Nat Rev Neurol. 2019; 15: 148-160.

25. Baizabal-Carvallo JF, Jankovic J. Movement disorders induced by deep brain stimulation. Parkinsonism Relat Disord. 2016; 25: 1-9.

26. Zarzycki MZ, Domitrz I. Stimulation-induced side effects after deep brain stimulation-a systematic review. Acta Neuropsychiatr. 2020; 32: 57-64.

27. Goedert M, Jakes R, Spillantini MG. The synucleinopathies: Twenty years on. J Parkinsons Dis. 2017; 7: S51-S69.

28. Carlson KW, Jason B, Socrates D, Shils JL, Mei L, Arle JE. Simulation: Critical ingredient of the electroceutical paradigm. Proceedings of the 40th Annual International Conference of the IEEE Engineering in Medicine and Biology Society; 2018 July 19th; Honolulu, USA. Piscataway Township: Institute of Electrical and Electronics Engineers.

29. Carlson KW, Shils JL, Mei L, Arle JE. Functional requirements of small- and large-scale neural circuitry connectome models. In: Brain and human body modeling 2020: Computational human models presented at IEEE EMBC 2019 and the brain initiative 2019 meeting. Cham: Springer Nature; 2020. pp.249-260.

30. Schroll $\mathrm{H}$, Hamker FH. Basal ganglia dysfunctions in movement disorders: What can be learned from computational simulations. Mov Disord. 2016; 31: 1591-1601.

31. Holt $A B$, Netoff TI. Computational modeling to advance deep brain stimulation for the treatment of Parkinson's disease. Drug Discov Today Dis Model. 2016; 19: 31-36.

32. Barbe MT, Reker P, Hamacher S, Franklin J, Kraus D, Dembek TA, et al. DBS of the PSA and the VIM in essential tremor: A randomized, double-blind, crossover trial. Neurology. 2018; 91: e543e550.

33. Castaneda E, Whishaw IQ, Robinson TE. Changes in striatal dopamine neurotransmission assessed with microdialysis following recovery from a bilateral 6-OHDA lesion: Variation as a function of lesion size. J Neurosci. 1990; 10: 1847-1854.

34. Bennett DA, Beckett LA, Murray AM, Shannon KM, Goetz CG, Pilgrim DM, et al. Prevalence of parkinsonian signs and associated mortality in a community population of older people. $\mathrm{N}$ Engl J Med. 1996; 334: 71-76.

35. Surmeier DJ. Determinants of dopaminergic neuron loss in Parkinson's disease. FEBS J. 2018; 285: 3657-3668.

36. Brown P. Cortical drives to human muscle: The piper and related rhythms. Prog Neurobiol. 2000; 60: 97-108.

37. Humphries MD, Obeso JA, Dreyer JK. Insights into Parkinson's disease from computational models of the basal ganglia. J Neurol Neurosurg Psychiatry. 2018; 89: 1181-1188.

38. DeLong MR. Primate models of movement disorders of basal ganglia origin. Trends Neurosci. 1990; 13: 281-285.

39. Yager LM, Garcia AF, Wunsch AM, Ferguson SM. The ins and outs of the striatum: Role in drug addiction. Neuroscience. 2015; 301: 529-541.

40. Lindahl M, Kotaleski JH. Untangling basal ganglia network dynamics and function: Role of 
dopamine depletion and inhibition investigated in a spiking network model. Eneuro. 2016; 3: ENEURO.0156-16.2016.

41. Humphries MD, Stewart RD, Gurney KN. A physiologically plausible model of action selection and oscillatory activity in the basal ganglia. J Neurosci. 2006; 26: 12921-12942.

42. Deffains M, Iskhakova L, Katabi S, Haber SN, Israel Z, Bergman H. Subthalamic, not striatal, activity correlates with basal ganglia downstream activity in normal and parkinsonian monkeys. Elife. 2016; 5: e16443.

43. Berke JD. Functional properties of striatal fast-spiking interneurons. Front Syst Neurosci. 2011; 5: 45 .

44. Lee HJ, Weitz AJ, Bernal-Casas D, Duffy BA, Choy M, Kravitz AV, et al. Activation of direct and indirect pathway medium spiny neurons drives distinct brain-wide responses. Neuron. 2016; 91: 412-424.

45. Milardi D, Quartarone A, Bramanti A, Anastasi G, Bertino S, Basile GA, et al. The cortico-basal ganglia-cerebellar network: Past, present and future perspectives. Front Syst Neurosci. 2019; 13: 61.

46. Coudé $D$, Parent A, Parent M. Single-axon tracing of the corticosubthalamic hyperdirect pathway in primates. Brain Struct Funct. 2018; 223: 3959-3973.

47. Kumaravelu K, Oza CS, Behrend CE, Grill WM. Model-based deconstruction of cortical evoked potentials generated by subthalamic nucleus deep brain stimulation. J Neurophysiol. 2018; 120: 662-680.

48. Li Q, Qian ZM, Arbuthnott GW, Ke Y, Yung WH. Cortical effects of deep brain stimulation: Implications for pathogenesis and treatment of Parkinson disease. JAMA Neurol. 2014; 71: 100103.

49. Anderson RW, Farokhniaee A, Gunalan K, Howell B, Mclntyre CC. Action potential initiation, propagation, and cortical invasion in the hyperdirect pathway during subthalamic deep brain stimulation. Brain Stimul. 2018; 11: 1140-1150.

50. Johnson LA, Wang J, Nebeck SD, Zhang J, Johnson MD, Vitek JL. Direct activation of primary motor cortex during subthalamic but not pallidal deep brain stimulation. J Neurosci. 2020; 40: 2166-2177.

51. Cury RG, Teixeira MJ, Galhardoni R, Silva V, Iglesio R, França C, et al. Connectivity patterns of subthalamic stimulation influence pain outcomes in Parkinson's disease. Front Neurol. 2020; 11: 9.

52. Shine JM, Bell PT, Matar E, Poldrack RA, Lewis SJ, Halliday GM, et al. Dopamine depletion alters macroscopic network dynamics in Parkinson's disease. Brain. 2019; 142: 1024-1034.

53. Ruppert MC, Greuel A, Tahmasian M, Schwartz F, Stürmer S, Maier F, et al. Network degeneration in Parkinson's disease: Multimodal imaging of nigro-striato-cortical dysfunction. Brain. 2020; 143: 944-959.

54. Rosenbaum R, Zimnik A, Zheng F, Turner RS, Alzheimer C, Doiron B, et al. Axonal and synaptic failure suppress the transfer of firing rate oscillations, synchrony and information during high frequency deep brain stimulation. Neurobiol Dis. 2014; 62: 86-99.

55. Grill WM, Snyder AN, Miocinovic S. Deep brain stimulation creates an informational lesion of the stimulated nucleus. Neuroreport. 2004; 15: 1137-1140.

56. Mclntyre CC, Anderson RW. Deep brain stimulation mechanisms: The control of network activity via neurochemistry modulation. J Neurochem. 2016; 139: 338-345. 
57. Stefani A, Trendafilov V, Liguori C, Fedele E, Galati S. Subthalamic nucleus deep brain stimulation on motor-symptoms of Parkinson's disease: Focus on neurochemistry. Prog Neurobiol. 2017; 151: 157-174.

58. Jakobs M, Fomenko A, Lozano AM, Kiening KL. Cellular, molecular, and clinical mechanisms of action of deep brain stimulation-a systematic review on established indications and outlook on future developments. EMBO Mol Med. 2019; 11: e9575.

59. Holt $A B$, Netoff TI. Computational modeling of epilepsy for an experimental neurologist. Exp Neurol. 2013; 244: 75-86.

60. Bijsterbosch J, Smith SM, Beckmann CF. Introduction to resting state fMRI functional connectivity. Oxford: OUP Oxford; 2017.

61. Chen LM, Yang PF, Wang F, Mishra A, Shi Z, Wu R, et al. Biophysical and neural basis of resting state functional connectivity: Evidence from non-human primates. Magn Reson Imaging. 2017; 39: 71-81.

62. Shen HH. Core concept: Resting-state connectivity. Proc Natl Acad Sci U S A. 2015; 112: 1411514116.

63. Lempka SF, McIntyre CC, Kilgore KL, Machado AG. Computational analysis of kilohertz frequency spinal cord stimulation for chronic pain management. Anesthesiology. 2015; 122: 1362-1376.

64. McIntyre CC, Richardson AG, Grill WM. Modeling the excitability of mammalian nerve fibers: Influence of afterpotentials on the recovery cycle. J Neurophysiol. 2002; 87: 995-1006.

65. Liu H, Roppolo JR, de Groat WC, Tai C. The role of slow potassium current in nerve conduction block induced by high-frequency biphasic electrical current. IEEE Trans Biomed Eng. 2009; 56: 137-146.

66. Ackermann DM, Bhadra N, Gerges M, Thomas PJ. Dynamics and sensitivity analysis of highfrequency conduction block. J Neural Eng. 2011; 8: 065007.

67. Arle JE, Mei L, Carlson KW, Shils JL. High-frequency stimulation of dorsal column axons: Potential underlying mechanism of paresthesia-free neuropathic pain relief. Neuromodulation. 2016; 19: 385-397.

68. Alqahtani A, Al Abed A, Lovell NH, Dokos S. Optimizing stimulation strategies for retinal electrical stimulation: A modelling study. Proceedings of the 2019 41st Annual International Conference of the IEEE Engineering in Medicine and Biology Society; 2019 July 23rd; Berlin, Germany. Piscataway Township: Institute of Electrical and Electronics Engineers.

69. Guo Z, Feng Z, Wang Y, Wei X. Simulation study of intermittent axonal block and desynchronization effect induced by high-frequency stimulation of electrical pulses. Front Neurosci. 2018; 12: 858.

70. Guo T, Tsai D, Yang CY, Al Abed A, Twyford P, Fried SI, et al. Mediating retinal ganglion cell spike rates using high-frequency electrical stimulation. Front Neurosci. 2019; 13: 413.

71. Muralidharan M, Guo T, Shivdasani MN, Tsai D, Fried S, Li L, et al. Neural activity of functionally different retinal ganglion cells can be robustly modulated by high-rate electrical pulse trains. J Neural Eng. 2020; 17: 045013.

72. Yang CY, Tsai D, Guo T, Dokos S, Suaning GJ, Morley JW, et al. Differential electrical responses in retinal ganglion cell subtypes: Effects of synaptic blockade and stimulating electrode location. J Neural Eng. 2018; 15: 046020.

73. Howells J, Trevillion L, Bostock H, Burke D. The voltage dependence of I(h) in human myelinated axons. J Physiol. 2012; 590: 1625-1640. 
74. Burke D, Howells J, Trevillion L, McNulty PA, Jankelowitz SK, Kiernan MC. Threshold behaviour of human axons explored using subthreshold perturbations to membrane potential. J Physiol. 2009; 587: 491-504.

75. Schwarz JR, Reid G, Bostock H. Action potentials and membrane currents in the human node of Ranvier. Pflügers Archiv. 1995; 430: 283-292.

76. Arle JE, Mei L, Carlson KW. Fiber threshold accommodation as a mechanism of burst and highfrequency spinal cord stimulation. Neuromodulation. 2020; 23: 582-593.

77. Gunalan K, Howell B, Mclntyre CC. Quantifying axonal responses in patient-specific models of subthalamic deep brain stimulation. Neurolmage. 2018; 172: 263-277.

78. Coombes S. Neural fields. San Diego: Brain Corporation; 2006. pp.1363.

79. Deco G, Jirsa VK, Robinson PA, Breakspear M, Friston K. The dynamic brain: From spiking neurons to neural masses and cortical fields. PLoS Comput Biol. 2008; 4: e1000092.

80. Kumaravelu K, Brocker DT, Grill WM. A biophysical model of the cortex-basal ganglia-thalamus network in the 6-OHDA lesioned rat model of Parkinson's disease. J Comput Neurosci. 2016; 40: 207-229.

81. Butson CR, McIntyre CC. Role of electrode design on the volume of tissue activated during deep brain stimulation. J Neural Eng. 2006; 3: 1-8.

82. Chaturvedi A, Lujan JL, McIntyre CC. Artificial neural network based characterization of the volume of tissue activated during deep brain stimulation. J Neural Eng. 2013; 10: 056023.

83. Rattay F. Analysis of models for extracellular fiber stimulation. IEEE Trans Biomed Eng. 1989; 36: 676-682.

84. Rattay F. The basic mechanism for the electrical stimulation of the nervous system. Neuroscience. 1999; 89: 335-346.

85. Rattay F. Analysis of models for external stimulation of axons. IEEE Trans Biomed Eng. 1986; 33: 974-977.

86. Rattay F. Analysis of the electrical excitation of CNS neurons. IEEE Trans Biomed Eng. 1998; 45: 766-772.

87. McNeal DR. Analysis of a model for excitation of myelinated nerve. IEEE Trans Biomed Eng. 1976; 23: 329-337.

88. Warman EN, Grill WM, Durand D. Modeling the effects of electric fields on nerve fibers: Determination of excitation thresholds. IEEE Trans Biomed Eng. 1992; 39: 1244-1254.

89. Moffitt MA, McIntyre CC, Grill WM. Prediction of myelinated nerve fiber stimulation thresholds: Limitations of linear models. IEEE Trans Biomed Eng. 2004; 51: 229-236.

90. Arle JE, Carlson KW, Mei L, Shils JL. Modeling effects of scar on patterns of dorsal column stimulation. Neuromodulation. 2014; 17: 320-333.

91. Peterson EJ, Izad O, Tyler DJ. Predicting myelinated axon activation using spatial characteristics of the extracellular field. J Neural Eng. 2011; 8: 046030.

92. van Albada SJ, Robinson PA. Mean-field modeling of the basal ganglia-thalamocortical system. I: Firing rates in healthy and parkinsonian states. J Theor Biol. 2009; 257: 642-663.

93. Gunalan K, Mclntyre CC. Biophysical reconstruction of the signal conduction underlying shortlatency cortical evoked potentials generated by subthalamic deep brain stimulation. Clin Neurophysiol. 2020; 131: 542-547.

94. Geddes LA. Accuracy limitations of chronaxie values. IEEE Trans Biomed Eng. 2004; 51: 176-181.

95. Lempka SF, Howell B, Gunalan K, Machado AG, McIntyre CC. Characterization of the stimulus 
waveforms generated by implantable pulse generators for deep brain stimulation. Clin Neurophysiol. 2018; 129: 731-742.

96. Grill WM, Mortimer JT. Electrical properties of implant encapsulation tissue. Ann Biomed Eng. 1994; 22: 23-33.

97. Washburn S, Catlin R, Bethel K, Canlas B. Patient-perceived differences between constant current and constant voltage spinal cord stimulation systems. Neuromodulation. $2014 ; 17$ : 2836.

98. Izhikevich EM. Hybrid spiking models. Philos Trans A Math Phys Eng Sci. 2010; 368: 5061-5070.

99. Thibeault CM, Srinivasa N. Using a hybrid neuron in physiologically inspired models of the basal ganglia. Front Comput Neurosci. 2013; 7: 88.

100. Brette R, Gerstner W. Adaptive exponential integrate-and-fire model as an effective description of neuronal activity. J Neurophysiol. 2005; 94: 3637-3642.

101. Arle JE, Mei LZ, Shils JL. Modeling Parkinsonian circuitry and the DBS electrode. Stereotact Funct Neurosurg. 2008; 86: 1-15.

102. Shils JL, Mei LZ, Arle JE. Modeling parkinsonian circuitry and the DBS electrode. II. Evaluation of a computer simulation model of the basal ganglia with and without subthalamic nucleus stimulation. Stereotact Funct Neurosurg. 2008; 86: 16-29.

103. Arle JE, Iftimia N, Shils JL, Mei L, Carlson KW. Dynamic computational model of the human spinal cord connectome. Neural Comput. 2019; 31: 388-416.

104. MacGregor RJ. Theoretical mechanics of biological neural networks. Boston: Academic Press; 1993.

105. Rall W. Electrophysiology of a dendritic neuron model. Biophys J. 1962; 2: 145-167.

106.Kang G, Lowery MM. Interaction of oscillations, and their suppression via deep brain stimulation, in a model of the cortico-basal ganglia network. IEEE Trans Neural Syst Rehabilitation Eng. 2013; 21: 244-253.

107.Kang G, Lowery MM. Effects of antidromic and orthodromic activation of STN afferent axons during DBS in Parkinson's disease: A simulation study. Front Comput Neurosci. 2014; 8: 32.

108. Dokos S. Modelling organs, tissues, cells and devices using MATLAB and COMSOL multiphysics. Berlin: Springer; 2017.

109. Yousif N, Mace M, Pavese N, Borisyuk R, Nandi D, Bain P. A network model of local field potential activity in essential tremor and the impact of deep brain stimulation. PLoS Comput Biol. 2017; 13: e1005326.

110.Otsuka T, Abe T, Tsukagawa T, Song WJ. Conductance-based model of the voltage-dependent generation of a plateau potential in subthalamic neurons. J Neurophysiol. 2004; 92: 255-264.

111.Rubin JE, Terman D. High frequency stimulation of the subthalamic nucleus eliminates pathological thalamic rhythmicity in a computational model. J Comput Neurosci. 2004; 16: 211235.

112. Arle JE, Mei LZ, Carlson KW, Shils JL. Theoretical effect of DBS on axonal fibers of passage: Firing rates, entropy, and information content. Stereotact Funct Neurosurg. 2018; 96: 1-12.

113.Johnson MD, Mclntyre CC. Quantifying the neural elements activated and inhibited by globus pallidus deep brain stimulation. J Neurophysiol. 2008; 100: 2549-2563.

114. Mclntyre CC, Grill WM. Extracellular stimulation of central neurons: Influence of stimulus waveform and frequency on neuronal output. J Neurophysiol. 2002; 88: 1592-1604.

115. Yousif N, Purswani N, Bayford R, Nandi D, Bain P, Liu X. Evaluating the impact of the deep brain 
stimulation induced electric field on subthalamic neurons: A computational modelling study. J Neurosci Methods. 2010; 188: 105-112.

116. Wang Y, Hutchings F, Kaiser M. Computational modeling of neurostimulation in brain diseases. Prog Brain Res. 2015; 222: 191-228.

117. Kuo CH, White-Dzuro GA, Ko AL. Approaches to closed-loop deep brain stimulation for movement disorders. Neurosurgical Focus. 2018; 45: E2.

118. Yao L, Brown P, Shoaran M. Improved detection of Parkinsonian resting tremor with feature engineering and Kalman filtering. Clin Neurophysiol. 2020; 131: 274-284.

119. Yu Y, Hao Y, Wang Q. Model-based optimized phase-deviation deep brain stimulation for Parkinson's disease. Neural Netw. 2020; 122: 308-319.

120.Pina-Fuentes D, Beudel M, Little S, Brown P, Oterdoom DLM, van Dijk JM. Adaptive deep brain stimulation as advanced Parkinson's disease treatment (ADAPT study): Protocol for a pseudorandomised clinical study. BMJ Open. 2019; 9: e029652.

121. Hirschmann J, Schoffelen JM, Schnitzler A, van Gerven MA. Parkinsonian rest tremor can be detected accurately based on neuronal oscillations recorded from the subthalamic nucleus. Clin Neurophysiol. 2017; 128: 2029-2036.

122. Hirschmann J, Schoffelen JM, Schnitzler A, van Gerven MA. Inferring the presence of Parkinsonian rest tremor from subthalamic local field potential recordings. Mov Disord. 2017; 32: S290.

123.Johnson LA, Nebeck SD, Muralidharan A, Johnson MD, Baker KB, Vitek JL. Closed-loop deep brain stimulation effects on Parkinsonian motor symptoms in a non-human primate-is beta enough? Brain Stimul. 2016; 9: 892-896.

124.Schiff SJ. Neural control engineering: The emerging intersection between control theory and neuroscience. Cambridge: MIT Press; 2012.

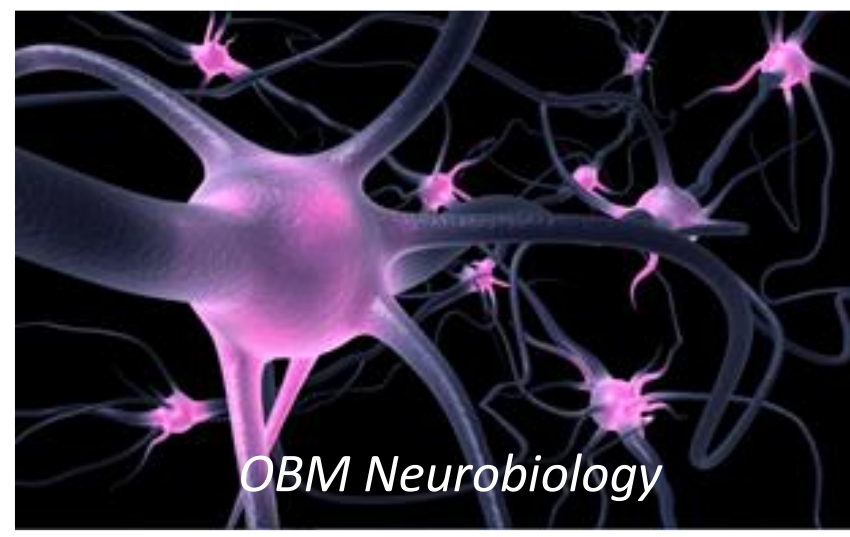

Enjoy OBM Neurobiology by:

1. Submitting a manuscript

2. Joining volunteer reviewer bank

3. Joining Editorial Board

4. Guest editing a special issue

For more details, please visit:

http://www.lidsen.com/journals/neurobiology 